\title{
Circuit
}

Musiques contemporaines

\section{Ragnar Kjartansson au Musée d'art contemporain de Montréal (MACM)}

\section{Simon Gervais}

Volume 26, numéro 3, 2016

URI : https://id.erudit.org/iderudit/1038522ar

DOI : https://doi.org/10.7202/1038522ar

Aller au sommaire du numéro

Éditeur(s)

Les Presses de l’Université de Montréal

ISSN

1183-1693 (imprimé)

1488-9692 (numérique)

Découvrir la revue

Citer ce document

Gervais, S. (2016). Ragnar Kjartansson au Musée d'art contemporain de

Montréal (MACM). Circuit, 26(3), 95-98. https://doi.org/10.7202/1038522ar d'utilisation que vous pouvez consulter en ligne.

https://apropos.erudit.org/fr/usagers/politique-dutilisation/ 


\section{Ragnar Kjartansson au Musée d’art contemporain de Montréal (MACM)}

Simon Gervais

Pour sa première exposition canadienne d'envergure, l'artiste multidisciplinaire islandais Ragnar Kjartansson ${ }^{1}$ venait présenter trois œuvres majeures de son corpus au MACM $^{2}$. Reconnu pour ses performances spectaculaires de très longue durée et au caractère répétitif qui oscille entre le tragique et le comique, le travail artistique de Kjartansson combine la vidéo, la performance, les arts visuels, la musique, et explore les limites physiques et psychologiques de l'artiste et de ses multiples représentations. Les trois œuvres présentées au MACM, en continuité avec ses explorations, posaient aussi des questions sur les rapports musico-visuels.

\section{A Lot of Sorrow, vidéo à canal unique, 2013, $369 \mathrm{~min}$}

Une musique rock à la mélodie envoûtante et mélancolique se fait entendre dans le corridor qui mène à l'exposition de l'artiste islandais. Déjà, la première des trois œuvres nous entraîne vers elle, sa musique outrepassant les limites des pièces de l'exposition, et oriente le parcours de la visite.

Projeté sur un gigantesque écran et un puissant système audio 2.1, A Lot of Sorrow est une captation vidéo du groupe The National ${ }^{3}$ performant leur chanson "Sorrow ${ }^{4}$ », d'une durée de 3 min 25 s, de manière continue et répétitive sur une période de six heures, c'est-à-dire 105 fois. La performance, filmée comme on filme des concerts pour les chaînes MTV ou VH1 donne à voir l'espace d'une salle de concert géné- rique. À première vue, la captation standardisée du concert et la répétition démesurée, thème récurrent chez Kjartansson, laissent entrevoir une volonté de banalisation de la performance en direct; il n'en est rien, du moins, pas dans notre espace spectatoriel.

"Suffit-il de voir un musicien jouer pour entrer dans sa musique ou la faire rayonner? Non », répond Jean-Louis Comolli ${ }^{6}$, et Kjartansson lui donne raison. Rapidement, à l'image, on constate que la répétition épuise les musiciens filmés: leur performance en est affectée, les marques de fatigue s'impriment sur l'écran. Cela appartient toutefois uniquement à cet espace. La répétition agit autrement sur le spectateur qui, pris dans la redite, peut s'abandonner et approcher un état de transe, c'est-à-dire (re)vivre le sentiment d'une performance en direct. L'image n'arrive pas à nous garder à distance de la musique ni à dominer le rapport audiovisuel.

Le dispositif de projection participe même de cette mise à distance. Pour accommoder un écran si grand, les enceintes acoustiques se voient « repoussées ${ }^{7}$ » sur les côtés de la salle. Il se produit alors un phénomène acoustique qui crée un vide sonore dans l’image. La musique, entendue clairement des côtés de la salle, n'émane pas de l'image et ne connecte pas avec celleci. La musique s'excentre du corps filmé qui la produit: l'image n'est pas ce qui envoûte, l'image n'est pas ce qui compte, c'est la musique qui est reine et souveraine. 
Par une répétition démesurée, combinée avec un dispositif particulier de projection audiovisuelle, Kjartansson fait émerger l'aspect live d'une performance à même un enregistrement audiovisuel et restitue à la musique une certaine souveraineté.

FIGURE 1 Extrait de l'installation A Lot of Sorrow au MACM. Crédit photo: Simon Gervais.

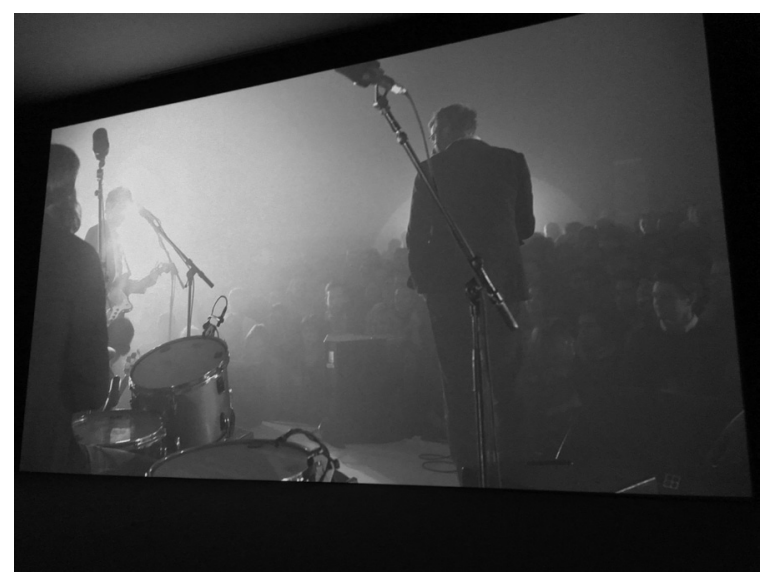

World Light - The Life and Death of an Artist, projection vidéo à quatre canaux, couleur, son, 2015

Si le dispositif de projection dans A Lot of Sorrow aide à rendre saillante une séparation, la contribution du dispositif dans World Light est plutôt de l'ordre de la confusion.

World Light est une adaptation filmique de l'œuvre littéraire du même nom, écrite par l'auteur islandais Halldór Laxness 8 . Ce récit étant constitué de quatre livres, Kjartansson a décidé de déployer son adaptation sur autant d'écrans; chacun étant doté d'une enceinte acoustique qui diffuse le son synchrone, ils sont répartis en croix dans la salle d'exposition, obligeant le spectateur à se placer au centre de l'espace.

Toutefois, ce ne sont pas les films qui sont projetés simultanément, mais l'entièreté du tournage requis

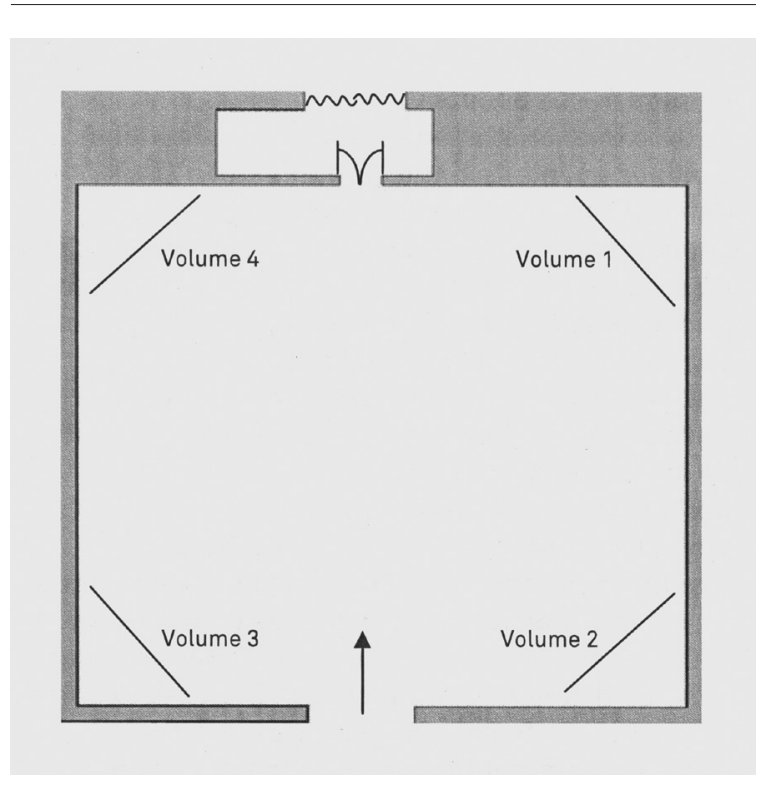

pour adapter les quatre livres: multiples plans et prises comprenant les erreurs de jeu ou de technique, cohabitation des comédiens, du narrateur (interprété par Kjartansson), de l'équipe technique et des musiciens, etc. Ce dispositif ne génère rien d'autre qu'un grand bruit, une sorte de chaos.

Si nous percevons globalement toujours l'idée de fragmentation et de déconnexion, nous sommes surtout confrontés à nos propres incapacités: on ne peut comprendre (le sens de l'œuvre nous échappe), on ne peut suivre (le fil narratif et la chronologie des événements d'une œuvre si dense sont impossibles à recomposer), et le caractère répétitif et ennuyeux des multiples prises faites pour capter un plan exagère ces contraintes. L'expérience de cette installation s'avère alors peu concluante. 
The Visitors, projection vidéo à neuf canaux, couleur, son, 2012, $64 \mathrm{~min}$

Derrière des portes closes se trouvant à même l'installation World Light, la troisième et dernière installation, gardée tel un secret, semble volontairement placée en fin de parcours. Clou de l'exposition, The Visitors est une performance musicale audiovisuelle répartie sur neuf écrans possédant chacun leur enceinte acoustique - sept écrans installés sur les quatre murs et deux écrans suspendus dans le milieu de la pièce se faisant dos. Huit écrans présentent chacun des musiciens. Ceux-ci sont isolés les uns des autres dans les nombreuses pièces d'un manoir, mais unis pour interpréter l'accrocheuse pièce musicale Feminine Ways inspirée d'un poème d'Ásdis Sif Gunnarsdóttir, l'ex-femme de l'artiste islandais. Le neuvième écran montre, en plan large, des amis de l'artiste. Ces derniers, situés à l'extérieur du manoir, écoutent la performance et produisent ici et là des sons. Tous dotés d'une paire d'écouteurs, les musiciens isolés sont reliés par l'écoute, par la musique, tandis que nous, visiteurs, les relierons de la même manière.

À notre arrivée dans la salle d'exposition, l'image et la musique nous interpellent chacune à sa manière. Le premier écran que l'on voit est celui du groupe d'amis, nous renvoyant déjà notre position de visiteur. La disposition des autres écrans rend impossible la vue de toutes les images en même temps et, par le fait même, de tous les musiciens. Seule la musique se révèle entièrement et englobe la salle elle est diffusée à un volume suffisamment élevé pour être entendue de partout dans cet espace. Toutefois, il est néces- saire de s'approcher près d'un écran si l'on veut capter les détails et nuances de chaque instrumentiste. Ainsi, bien que les musiciens soient tous réunis et le contexte donné, la musique reste à être entendue, ou plutôt mixée par le visiteur. Le déplacement et le (re)mixage sont, indissociablement, inévitables. Plus encore, ce dispositif audio-musico-visuel revisite l'idée du mixage.

Le contexte de l'œuvre rappelle déjà la manière dont les disques sont enregistrés de nos jours: des musiciens isolés qui enregistrent leur trame séparément pour conserver le plein contrôle sur chaque instrument lors du mixage ; l'occupation de lieux mythiques à la réverbération unique et singulière; l'aspect répétitif qui rappelle les nombreuses prises souvent requises pour obtenir la performance désirée. La dimension supplémentaire et nouvelle de cette œuvre est le mixage en temps réel; mais elle n'existe que parce que l'image a un pouvoir d'attraction qui nous oblige à constamment réentendre la musique. Chaque déplacement, chaque mouvement de la tête augmente ou diminue la

FIGURE 3 Extrait de l'installation The Visitors au maCM. Crédit photo: Simon Gervais.

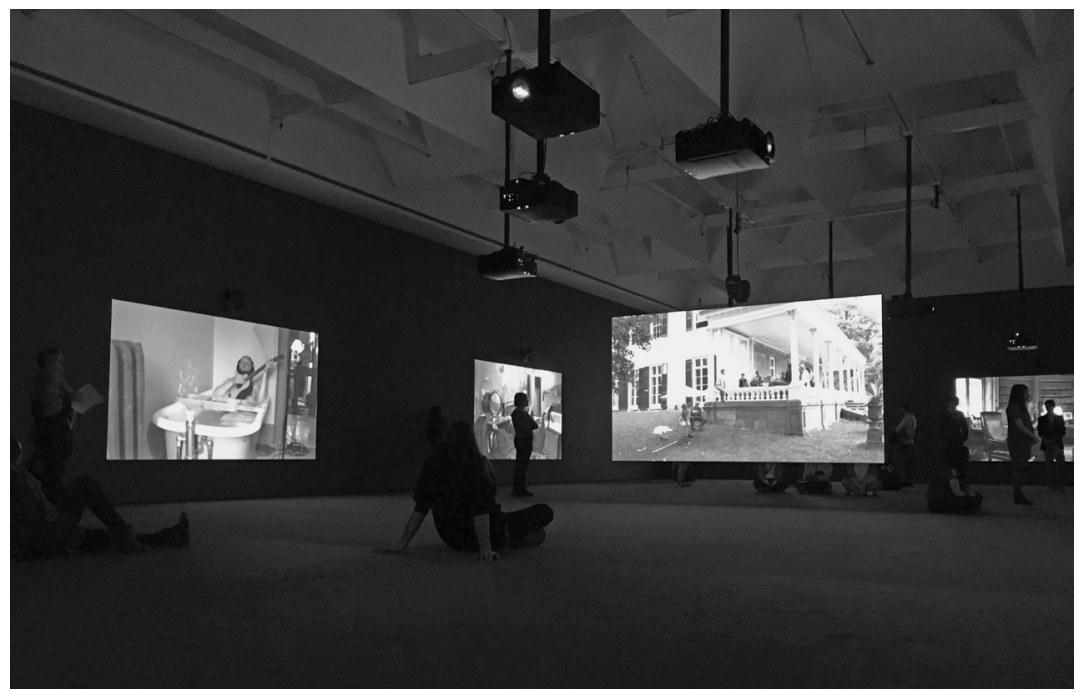


réverbération, modifie la spatialisation des instruments dans notre stéréophonie personnelle: c'est, ainsi, un mixage à part entière. La durée et le caractère répétitif ne sont pas étrangers à ce mode d'expérience de la musique; ces aspects donnent le temps au visiteur d'apprécier chaque instrumentiste, chaque nuance de la performance, mais surtout, chaque nuance de son propre mixage.

D’un même élan, la musique de chaque instrument ne parvient à s'élever au-dessus de la mêlée que si elle prend corps littéralement. Pour que notre regard se pose longuement sur un écran et qu'émerge seul l'instrument désiré, Kjartansson articule notre fascination pour ces corps musicaux cadrés de manière précise, concentrés et fébriles dans leur écoute des autres. «Filmer l'écoute. Il y a peut-être plus de musique dans le visage filmé de celui qui écoute que de celui qui joue ${ }^{9}$.»

La finale de l'œuvre est, en ce sens, révélatrice de cet aspect: l'un après l'autre, chaque musicien quitte son poste, abandonnant son instrument sur place, allant en rejoindre un autre au passage, avec pour objectif que tous aboutissent à l'extérieur du manoir et prennent la clé des champs en chantant à l'unisson l'inlassable refrain. Les visiteurs de l'exposition font de même, les suivant d'écran en écran, toujours envoûtés par la mélodie de Kjartansson, pour tous se regrouper devant l'écran qui montre l'extérieur du manoir. Les caméras, elles, continuent de filmer les pièces inhabitées et les instruments abandonnés. Sans musique, les images ne sont plus que des contenants vides. Sans musique, les images perdent leur pouvoir d'attraction, qu'elles soient diminuées par l'absence de corps musicaux dans The Visitors, ou qu'elles aient été précédemment vidées par le gigantesque dispositif de A Lot of Sorrow.

Kjartansson montre, à travers sa musique filmée, que le caractère fascinant et attirant des images, appuyé par l'effet spectaculaire des écrans, est en réalité modulé par la musique. Toutefois, par cette modulation, la musique est contrainte de vider totalement l'image de son pouvoir d'attraction. L'égalité musico-visuelle est «impossible».

\footnotetext{
1. Pour plus d'informations sur Ragnar Kjartansson, voir : $<$ www.luhringaugustine.com/artists/ragnar-kjartansson $>$ (consulté le 27 septembre 2016).

2. Pour plus d'informations sur l'exposition, voir: <www.macm.org/ expositions/ragnar-kjartansson> (consulté le 27 septembre 2016).

3. Formé en 1999 à Brooklyn, The National est un groupe de musique "indie rock». Leurs chansons sont souvent qualifiées comme étant mélancoliques. Pour plus d'informations, voir : < http://americanmary. com> (consulté le 27 septembre 2016).
}

4. Cette pièce se retrouve sur l'album High Violet, paru en 2010 sur l'étiquette $4 \mathrm{AD}$.

5. Ce type de captation de spectacle combine certains codes du vidéoclip (montage rapide, cadres et prises de vue originales, mouvement constant de la caméra) et les codes habituels du direct (authenticité du spectacle, série de plans qui combinent la vue des interprètes et de la foule, etc.).

6. Jean-Louis Comolli (1996), Voir et Pouvoir, Paris, Verdier, p. 322.

7. Nous mettons le mot entre guillemets car nous sommes conscient qu'il s'agit d'un placement volontaire - d'autres placements auraient permis d'éviter le phénomène acoustique décrit. Par exemple, ce problème est évité dans les autres salles en plaçant l'enceinte au-dessus de l'écran.

8. Heimsljós (1937, 1938, 1939, 1940), basé sur l'histoire du poète islandais de la fin du xıxe siècle Magnus Hjaltason Magnusson, est considéré comme l'œuvre la plus importante de Halldór Laxness.

9. Comolli, op. cit., p. 323. 